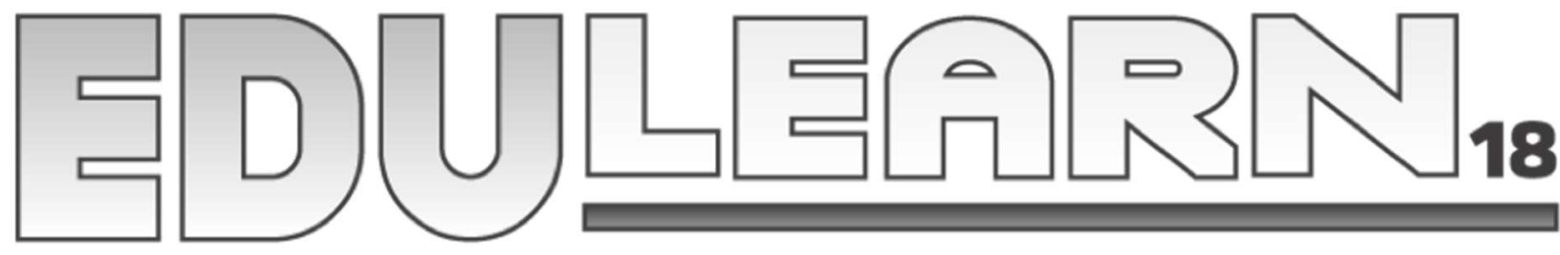

\title{
TEMPORALITY MATTERS. A LEARNING ANALYTICS STUDY OF THE PATTERNS OF INTERACTIONS AND ITS RELATION TO PERFORMANCE
}

\author{
M. Saqr, J. Nouri, U. Fors \\ Stockholm University (SWEDEN)
}

\begin{abstract}
Although temporality is embodied in instructional design, implicitly present in several learning theories and central to the self-regulation of learning and awarding of credits, it has not received the due attention in the field education. This learning analytics study included four higher education courses in dental education over a full year duration. Temporality in terms of when students engage in learning was studied on daily, weekly, course, and year basis. The patterns of low and high achiever groups in each period were visually plotted and compared. Correlation with the performance was evaluated using the non-parametric Spearman correlation test using re-sampling permutation technique. The findings of this study highlight some important points; temporality is a defining factor of how students regulate their learning and should be taken into account when designing a possible monitoring system. High achievers were always active early in the year, in the course, and on assignments. Low achievers, on the other hand, tend to be significantly more active close to examination times. Using only temporality predictors, we were able to predict high achievers with $100 \%$ precision and low achievers with $82.3 \%$ to $93.3 \%$ class precision. Since early participation was the predictor, it means that an early alert indicator can be achieved that enables timely intervention.
\end{abstract}

Keywords: Learning analytics, temporality, collaborative learning, time, procrastination, collaborative learning, problem-based learning

\section{INTRODUCTION}

Human beings have to follow the nature's unidirectional, uniform, and inexorable law of time, and so does every natural phenomenon. Learning is no exception; it is heavily influenced by time over many levels. The lecture and learning sessions of all kinds are timed, learning tasks and assignments are bound by deadlines, etc., crossing these boundaries have known consequences. Regulation extends to courses, academic years, degrees and academic cycles [1, 2]. Physiologically, attention span, cognitive capacity, memory and our human bodies have an internal clock that coordinates their function. As such, the time construct and temporality are fundamental elements in learning and teaching [3]. Therefore, time has been implicit in learning theories. An example is the self-regulation theory. When a student approaches a learning task, the student researches the aspects of task regarding constraints and affordances in terms of capacity and time. Self-regulation involves research and planning, engagement with the task, evaluation of performance and re-adjustment. All such elements are regulated by time [2, 4]. Unfortunately, the temporal aspects of learning have to a large degree been overlooked by research, partly because of methodological challenges and lack of data.

However, as a consequence of the digitalization of learning, large amounts of data has been materialized and made available for analysis. To harness the potential value of this data, learning analytics has emerged as a research discipline that analyze and explore the large amounts of data 
recorded in different digital environments [5]. Using predictive models, researchers were able to predict underachievers, find ways to inform teachers about instructional design and create meaningful interventions [6]. Nevertheless, concerns remain regarding the reproducibility and generalizability of the predictive models. Many reasons have been suggested, for instance, lack of theoretical underpinning, contextual factors, cultural differences and the element of temporality [7, 8]. In particular, the temporality dimension has not received due attention while being an important factor of regulation of learning.

Previous research has mostly considered the task-on-time as a variable, but results have been disappointing $[7,9,10]$. A recent study compared different ways to model the time-on-task and concluded that it should not be considered a reliable factor until we reach an agreeable standard measurement method [9]. Nonetheless, a broad classification can be reasonably made based on the levels at which time is regulated in education; namely a micro level which includes the short time intervals such as session time, task time as well as day and week activity measures; Meso level, which includes courses and curricula; and macro level that includes the whole academic year [1].

We argue here that the study of time and temporality factors might offer a clue to why we sometimes fail to predict students' behavior. Furthermore, when we are able to reach an accurate model, the model fails to generalize and work in comparable contexts. This study aims at study the patterns of students' interactions across the temporal dimensions of day, week, course, and academic year. A particular emphasis will be placed on the differences between high achievers and low achievers to see if either group has a distinct pattern.

\section{METHODOLOGY}

\section{The context}

The study included four courses in the College of Dentistry, Qassim University and covered a full academic year. All the courses followed a problem-based learning approach. Each week, students have to work online collaboratively on a new online problem, typically in small groups with a moderator. The interactions occur in a computer supported collaborative environment (CSCL), where students interact asynchronously on their own pace at the time they prefer. The interactions are expected to stimulate previous knowledge, trigger critical thinking, and stimulate an interdependent cooperative discussion to reach the learning objectives and conclusions $[11,12]$.

\section{METHODOLOGY}

Data collection: The interaction data, timestamps, and user attributes were extracted from the Moodle learning management through the "ad-hoc database queries" plugin, the plugin is available from the Moodle plugin directory. The data retrieved included ID of each user, group membership, course enrollments, each interaction made, time, target of the interaction and subject of interaction [13].

Data processing: Data were inspected and explored for corrupted, missing, or incomplete records. A single corrupt observation was removed due to absence of information. Collected data were combined with performance in a single table. IDs and identifying information were removed to completely anonymize the data. We then classified students based on final course grade to high achievers (top $50 \%$ of the students) and low achievers (bottom $50 \%$ of the class). For each user we calculated the number of interactions in each day, week (early bird and late), course (early and late), and year. By "early bird" we mean interactions on the first working day of the week, and by early during the course we mean the interaction occurring in the first half of the course duration.

Data analysis: Interactions were plotted and compared for each group using R! Programming language version 3.4.3. The plots included daily, weekly, course and year wise interactions for each course. Correlation with the performance was performed with the non-parametric Spearman correlation coefficient using re-sampling permutation technique. The level of statistical significance was set at 0.05 . We used the predictive algorithm K-nearest neighbors algorithm (KNN) to classify students according to achievement level. To prevent overfitting and enhance the reproducibility of the model a 10-fold crossvalidation was performed. 


\section{RESEARCH ETHICS}

All personal data were anonymized and excluded from analysis. Approval of the study protocol, data collection methods and analysis were obtained from the Regional Research Ethics Committee of Qassim. The privacy guidelines of the university regarding data collection, anonymization, storage, handling and reporting. The authors of this research were not involved in any teaching or grading activity in the studied courses.

\section{RESULTS}

The study included 5,963 interactions by 215 students in four courses over the period from eighth of September to May 1 2015. Course I had 2,877 interactions over three months, Course II had 1,050 interactions over 48 days, Course III had 947 interactions over 50 days, and Course IV had 1,089 over 45 days.

\section{Day level}

Temporality over the course of the day has revealed that high and low achievers show a similar pattern of high activity during the after working hours (study time) and low activity during the working hours as shown in figure 1. However, in the four studied courses, high achievers (red) tended to work more in the working hours more than their counterparts did. We calculated the number of interaction by each student during the working hours and explored if it is correlated with performance in table 1 , the results showed that there was consistent positive and statistically significant correlation between grade and working during this period. An indication that students who post and interact during working hours are more likely to be achievers.
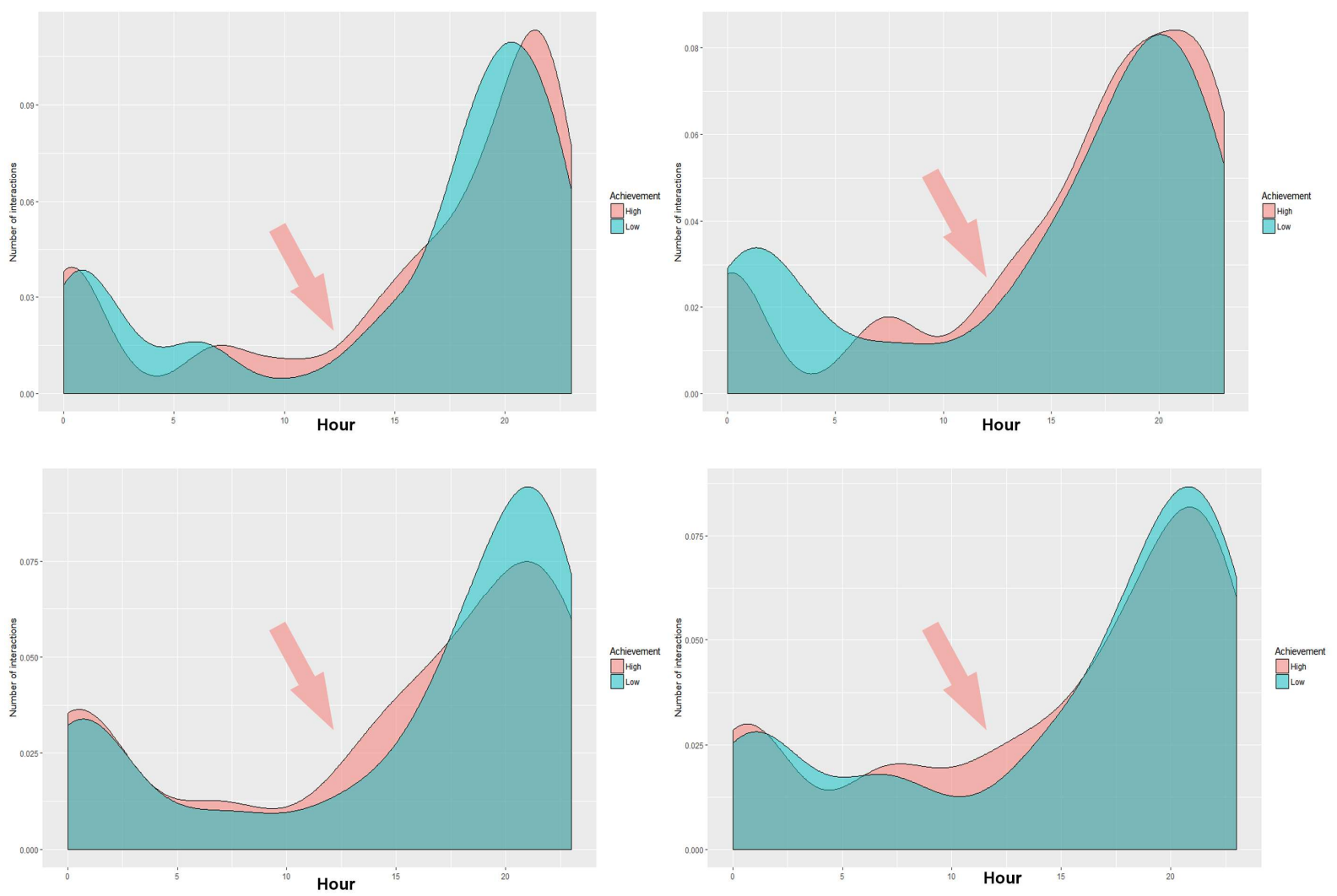

Figure 1 a density plot of activity during the 24 hours of the day in each course, high achievers (red), tend to work more during the hours from 7 AM to 4 PM (red arrows pointing).

Table 1 correlation between interactions during working hours and grade

\begin{tabular}{l|l|l|}
\hline Course & Spearman's rho Correlation coefficient $(r)$ & $P$
\end{tabular}




\begin{tabular}{|l|c|c|}
\hline Course I & 0.44 & $<0.01$ \\
\hline Course II & 0.32 & 0.02 \\
\hline Course III & 0.50 & $<0.01$ \\
\hline Course IV & 0.39 & $<0.01$ \\
\hline
\end{tabular}

\section{Week level:}

Temporal variations over the course of the week may be the most relevant to our study since the course is organized over a weekly basis. We calculated the number of early bird interactions for each student and compared both groups as displayed in figure two. The results showed that there is a clear and statistical significant difference between both groups (high achievers and low achievers) in terms of number of interactions in the beginning of the weeks. The difference was also consistent in each studied course. High achievers tend to interact more intensively early in the week.
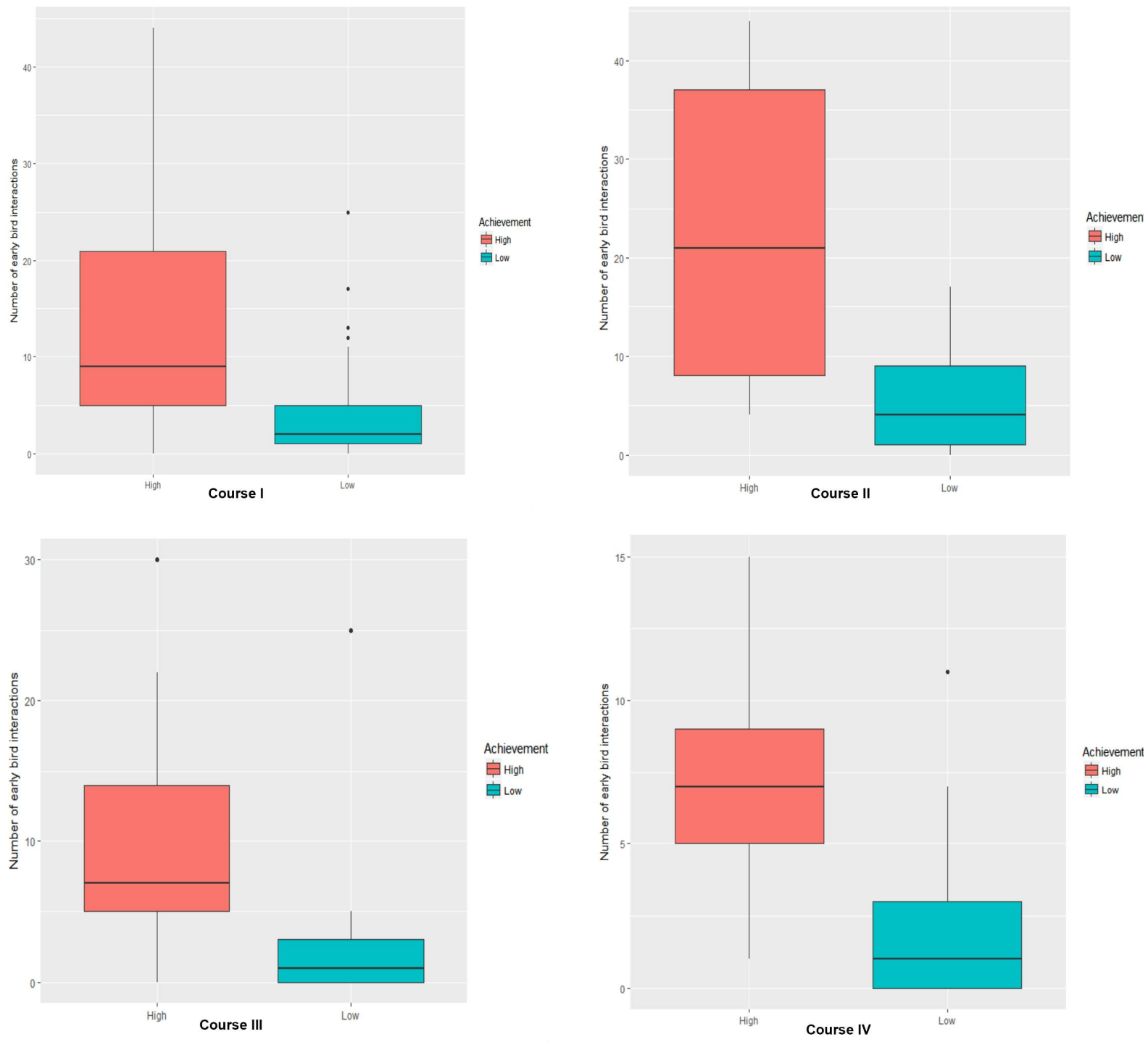

Figure 2 a boxplot comparison of early bird interactions in every course

Table 2 correlation between number of early bird interactions and grade

\begin{tabular}{|l|r|r|l|}
\hline Course & Spearman's rho Correlation coefficient $(\boldsymbol{r})$ & $\boldsymbol{P}$ & \\
\hline Course I & 0.48 & $<0.01$ \\
\hline Course II & 0.5 & $<0.01$ \\
\hline
\end{tabular}




\begin{tabular}{|l|r|r|}
\hline Course III & 0.4 & $<0.01$ \\
\hline Course IV & 0.55 & $<0.01$ \\
\hline
\end{tabular}

\section{Course level}

Similar to the pattern observed in the temporality on the week level, students who interacted more early during the course were more likely to be the high achievers, as seen in figure 3 the difference was consistent in each course. The correlation with performance was also statistically significant and ranged from moderate to strong. Full correlation data are presented in table 3.
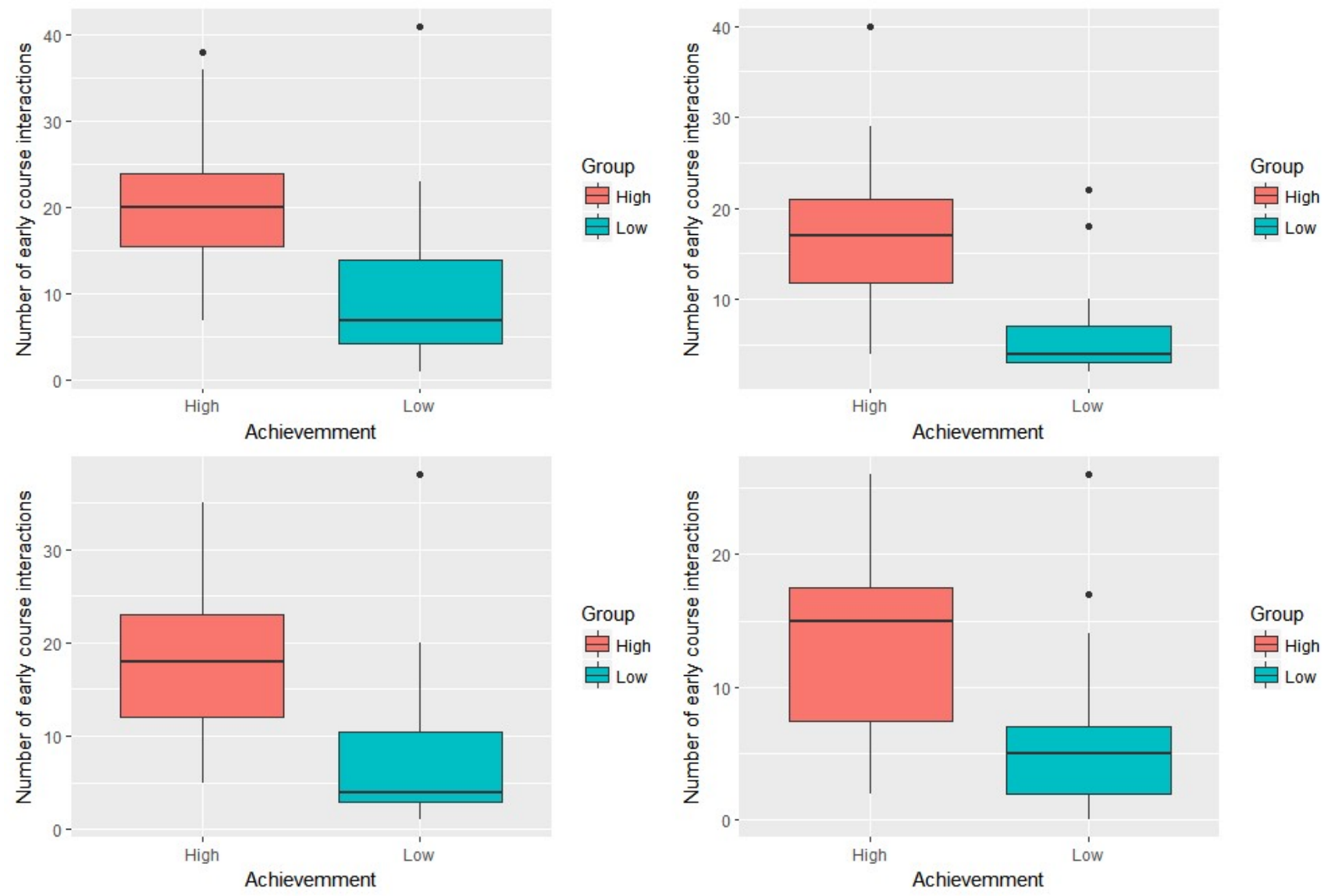

Figure 3 a boxplot comparison of early course interactions in every course

Table 3 correlation between the number of early course interactions and grade

\begin{tabular}{|l|c|c|}
\hline Course & Spearman's Rho Correlation coefficient $(\boldsymbol{r})$ & $\boldsymbol{P}$ \\
\hline Course I & 0.53 & $<0.01$ \\
\hline Course II & 0.7 & $<0.01$ \\
\hline Course III & $0.64^{*}$ & $<0.01$ \\
\hline Course IV & 0.58 & $<0.01$ \\
\hline
\end{tabular}

\section{Year level}

In contrast to the patterns observed earlier, there was a marked variation of the activity across the year level. High achievers were actively working hard during the early months of the year. The pattern was reversed during the last months of the year, and low achievers were the ones most active. It is obvious that yearlong temporality is not a reliable indicator of achievement.

\section{Can temporality predict performance?}


We then used the temporality variables, namely: number of interactions during working hours, early bird weekly interactions, and early course interactions in K-nearest neighbor's algorithm to predict high and low achievers. To prevent overfitting and improve the possibility of generalization and reproducibility of the model we used 10 -fold cross validation. The results presented in table 4 showed that temporality data can be a reasonably predictor of performance, where class precision of identifying low achievers ranged from $82.3 \%$ to $93.3 \%$ and $100 \%$ for high achievers.

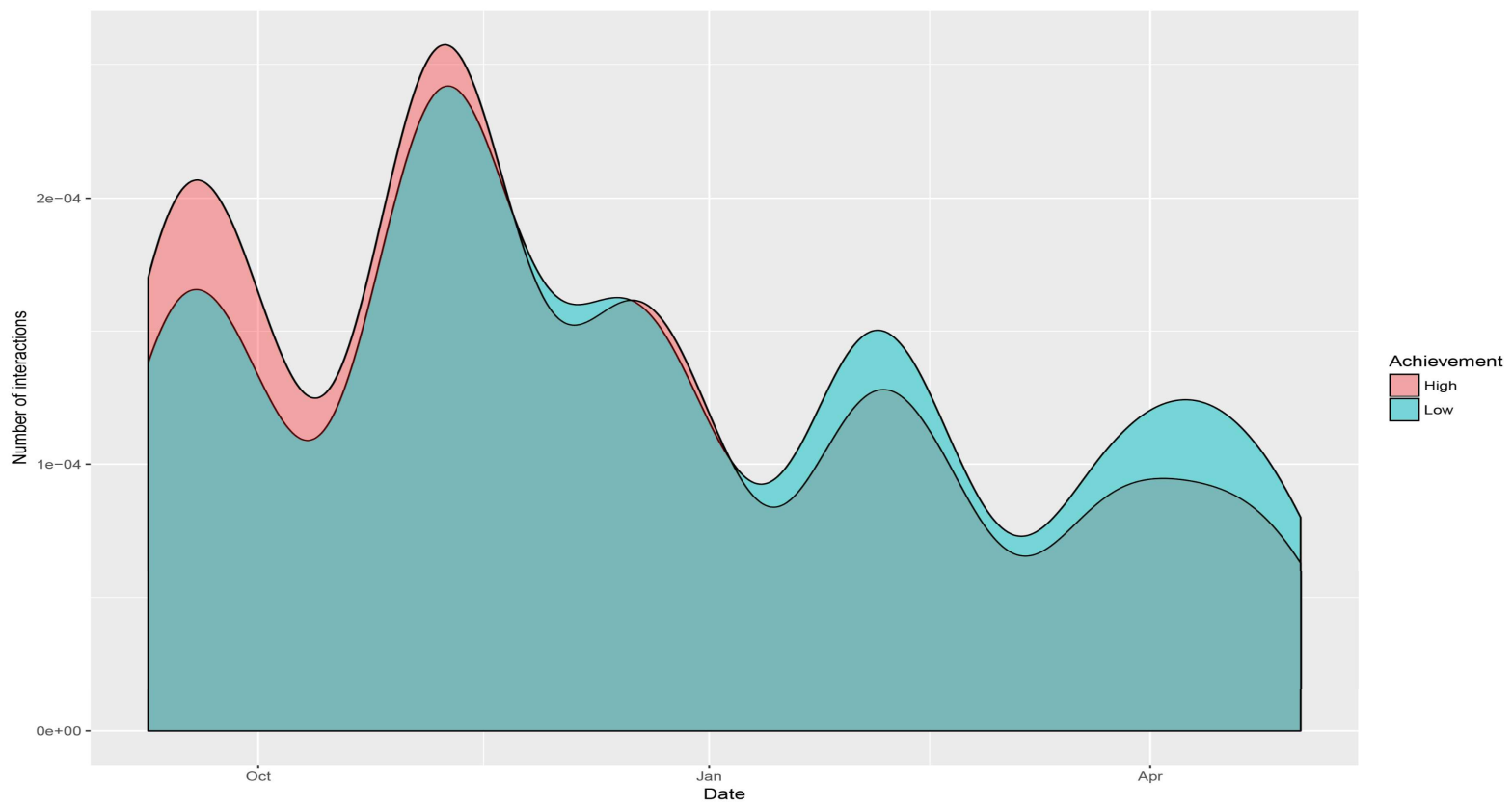

Figure 4 a density plot of activity during the whole year.

Table 4 A 10 fold cross-validated KNN algorithm used to predict high and low achievers in each course

\begin{tabular}{|c|c|c|c|}
\hline \multicolumn{4}{|c|}{ Course I } \\
\hline Course II & Actual Low & Actual High & Class precision \\
\hline Predicted Low & 28 & 6 & $82.4 \%$ \\
\hline Predicted High & 0 & 20 & $100.0 \%$ \\
\hline Class recall & $100.0 \%$ & $76.9 \%$ & \\
\hline \multicolumn{4}{|c|}{ Course II } \\
\hline Course III & Actual Low & Actual High & Class precision \\
\hline Predicted Low & 27 & 4 & $87.1 \%$ \\
\hline Predicted High & 0 & 23 & $100.0 \%$ \\
\hline Class recall & $100.0 \%$ & $85.2 \%$ & \\
\hline \multicolumn{4}{|c|}{ Course III } \\
\hline
\end{tabular}




\begin{tabular}{|r|c|c|c|}
\hline Course IV & Actual Low & Actual High & Class precision \\
\hline Predicted Low & 28 & 2 & $93.3 \%$ \\
\hline Predicted High & 0 & 23 & $100.0 \%$ \\
\hline Class recall & $100.0 \%$ & $92.0 \%$ & \\
\hline Course IV & Actual Low & Actual High & Class precision \\
\hline Predicted Low & 27 & 4 & $87.1 \%$ \\
\hline Class recall & $100.0 \%$ & $85.2 \%$ & $100.0 \%$ \\
\hline
\end{tabular}

The findings of the predictive algorithm highlight a very interesting finding that timing can be used with very good accuracy to predict students' achievement. Furthermore, since early participation is the predictor, it means that an early alert indicator can be achieved that enables timely intervention.

\section{DISCUSSION}

Previous research has focused on total counts of clicks, views, and sum of online activities. An approach that has been criticized for ignoring the time dimension that plays an important factor in how we teach and how students learn [1, 2]. This study was performed to investigate the patterns of temporality in online collaborative learning. The goal was to understand the different patterns of temporality beyond the session duration or the total duration of being online during a course. The study extended the analysis to multiple temporality levels and used visual insights as a guide to statistical analysis.

Timestamps are often abundant and might be overwhelming to analyze, however, visualization offers an excellent summarization function. A single graph enabled us to communicate thousands of interactions from hundreds of students over a year duration; it was helpful on course, week, and day level. Inspection of daily interactions graphs revealed an interesting pattern that would have been impossible to notice without visualization. The observed pattern was that high achievers tended to work online more than their counterparts do during working college hours, a finding that was consistent with each course. This pattern was later used successfully as a predictor of achievement. The same pattern was observed through visualization of early participation in the weekly activities and during the first half of the course.

Using only temporality indicators, the results of the KNN predictive algorithm were consistently reliable and accurate across every course. The algorithm tended to successfully classify high achievers better than low achievers. Nonetheless, the accuracy for identifying low achievers was still reasonable. Having indicators that have a high predictive value early in a course is a precious asset to educators who can tailor impactful support to their students based on such information.

Temporality is a defining factor of how students regulate their learning and should be taken into account when designing a learning analytics monitoring system. Educators and researcher can harness the power of time information readily available in learning management systems. Different approaches can be used to analyze such data to extract insights that can help us understand learning and learners' behavior. While, our study may be contextually limited, it offers a proof of concept of the value of the temporality that is applicable in a wide array of educational settings.

While the replication of our results is yet to be proven, we argue that the findings are less important than the approach. The approach is valid, relevant and has a considerable potential. Furthermore, the approach can be widely applied to different contexts and learning scenarios. We urge fellow researchers to build on our methods and create innovative ways to visualize different time patterns. There are many ways to analyze the time constructs such as time series analysis, survival analysis, and forecasting. Such methods can shed light on, for instance, engagement of students in online environments and drop out tendencies. 


\section{CONCLUSIONS}

The visualization of temporality was rather informative of students' online activity patterns. High achievers tended to work early during the week and course compared to their counterparts. Using only temporality indicators, we were able to predict students' achievement with a reasonable accuracy. Since early participation was the predictor, it means that an early alert indicator can be developed, which enables timely interventions, if needed. Thus, temporality is a defining factor of how students regulate their learning and should be taken into account when designing a possible learning analytics monitoring system for higher education.

\section{REFERENCES}

1. Barbera, E., B. Gros, and P. Kirschner, Paradox of time in research on educational technology. Time \& Society, 2015. 24(1): p. 96-108.

2. Knight, S., A.F. Wise, and B. Chen, Time for Change: Why Learning Analytics Needs Temporal Analysis. Journal of Learning Analytics, 2017. 4(3): p. 7-17.

3. Tonetti, L., V. Natale, and C. Randler, Association between circadian preference and academic achievement: a systematic review and meta-analysis. Chronobiology international, 2015. 32(6): p. 792-801.

4. Winne, P.H., A cognitive and metacognitive analysis of self-regulated learning. Handbook of self-regulation of learning and performance, 2011: p. 15-32.

5. Saqr, M., Learning analytics and medical education. International journal of health sciences, 2015. 9(4): p. 1-2.

6. Papamitsiou, Z. and A.A. Economides, Learning analytics and educational data mining in practice: A systematic literature review of empirical evidence. Journal of Educational Technology \& Society, 2014. 17(4): p. 49.

7. Saqr, M., U. Fors, and M. Tedre, How learning analytics can early predict under-achieving students in a blended medical education course. Medical Teacher, 2017. 39(7): p. 757--767.

8. Saqr, M., U. Fors, and M. Tedre, How the study of online collaborative learning can guide teachers and predict students' performance in a medical course. BMC Medical Education, 2018. 18(1): p. 1--14.

9. Kovanović, V., et al., Does time-on-task estimation matter? Implications for the validity of learning analytics findings. Journal of Learning Analytics, 2015. 2(3): p. 81--110.

10. Judd, T., Making sense of multitasking: The role of Facebook. Computers \& Education, 2014. 70: p. 194-202.

11. Mohamed Almohaimeed, I.A.R., Mohammed Saqr, E-Tutorial, an innovative and effective approach in PBL, in 6th International Conf on PBL in Dentistry. 2009: Hong Kong.

12. Wood, D.F., Problem based learning. BMJ, 2003. 326(7384): p. 328-30.

13. Hunt, T. and M. Kassaei. Reports: Ad-hoc database queries. [cited 2018 19-3-2018]; Available from: https://moodle.org/plugins/report_customsql. 